\title{
The NASA Juncture Flow Experiment: Goals, Progress, and Preliminary Testing (Invited)
}

\author{
Christopher L. Rumsey, Dan H. Neuhart ${ }^{\dagger}$ Michael A. Kegerise ${ }^{\ddagger}$ \\ NASA Langley Research Center, Hampton, VA 23681-2199
}

\begin{abstract}
NASA has been working toward designing and conducting a juncture flow experiment on a wing-body aircraft configuration. The experiment is planned to provide validation-quality data for CFD that focuses on the onset and progression of a separation bubble near the wing-body juncture trailing edge region. This paper describes the goals and purpose of the experiment. Although currently considered unreliable, preliminary CFD analyses of several different configurations are shown. These configurations have been subsequently tested in a series of "risk-reduction" wind tunnel tests, in order to help down-select to a final configuration that will attain the desired flow behavior. The risk-reduction testing at the higher Reynolds number has not yet been completed (at the time of this writing), but some results from one of the low-Reynolds-number experiments are shown.
\end{abstract}

\section{Introduction}

Geometric junctures (corners) are common features on most aerospace vehicles. Wing-body juncture flow is of particular interest to aircraft design engineers, because non-streamlined flow behavior such as separation bubbles in this area can have an adverse impact on aircraft performance. There is consequently a need to be able to accurately predict the behavior of the flow in juncture regions when using computational fluid dynamics (CFD).

In recent years, there has been significant confusion surrounding the computation of separated juncture flows. In the third Drag Prediction Workshop (DPW-3), ${ }^{1}$ which used the DLR-F6 wing-body geometry, participants predicted a very wide range of side-of-body separation bubble sizes with CFD. The same issue also came up for the Common Research Model (CRM) configuration at certain higher-than-cruise-angle-of-attack conditions in DPW-4 and DPW5. ${ }^{2}$ Many subsequent studies looked at the issue (see, e.g., Refs. 3-5); the computed juncture bubble appears to be influenced by a variety of factors, including grid size, grid topology, and numerical treatments. Because of this high degree of uncertainty in the CFD predictions, relevant separated corner flow experiments focused specifically on obtaining high-quality data for CFD validation are needed.

The flow physics of wing-body juncture flows is quite complex because it can involve several distinct vortical structures. ${ }^{6,7}$ Typically a large, unsteady horseshoe vortex as well as a smaller corner vortex are generated near the wing leading edge at the wing-body intersection, and wrap around the wing. There is also a smaller corner vortex initiated by gradients of the Reynolds stresses. ${ }^{8}$ Bordji et al. ${ }^{7}$ refer to this smaller vortex as a "stress-induced vortex." All of these vortices pass downstream, influencing the flow field in the juncture region. Although there is general awareness that the presence of a larger horseshoe vortex can help suppress corner separation, ${ }^{9}$ at the current time the precise manner in which the vortical structures and associated Reynolds stresses interact to cause and/or suppress corner separation is not fully understood. Also note that many factors-such as incoming boundary layer momentum deficit factor, wing bluntness factor, and wing sweep—-play some role in the juncture flow physics. ${ }^{10}$

Over time, the CFD community has come to the consensus that linear (Boussinesq) turbulence models such as Spalart-Allmaras (SA), ${ }^{11}$ Menter's shear stress transport (SST), ${ }^{12}$ and others will produce unrealistically large juncture separation bubbles when using finer grids and/or highly accurate numerics. In other words, when sufficiently reducing numerical discretization errors, a linear turbulence model will predict juncture separation bubbles inaccurately because of the construction of the turbulence model itself. Yamamoto et al. ${ }^{13}$ demonstrated that the use of the quadratic constitutive relation (QCR) of Spalart ${ }^{14}$ (which is a nonlinear fix that can be used in conjunction with any Boussinesq model) could improve corner flow CFD predictions for the CRM. Similarly, in Rumsey, ${ }^{15}$ SA-QCR as well as other

\footnotetext{
* Research Scientist, Computational AeroSciences Branch, Mail Stop 128, Fellow AIAA.

${ }^{\dagger}$ Research Scientist, Flow Physics and Control Branch, Mail Stop 170.

${ }^{\ddagger}$ Research Scientist, Flow Physics and Control Branch, Mail Stop 170, Senior Member AIAA.
} 
nonlinear models such as explicit algebraic stress and second-moment Reynolds-stress were shown to be qualitatively better than linear models for the CRM, in terms of predicting expected bubble size. Dandois ${ }^{16}$ used SA-QCR to demonstrate improved predictions for the DLR-F6 wing-body configuration as well as for a shock-induced separation in a rectangular duct. Presumably, the nonlinear models perform better because they more accurately represent the Reynolds stresses in the corner region. Gradients of the Reynolds stresses lead to secondary flow behavior ${ }^{17}$ that may play an important role in the formation of the stress-induced vortex, and consequently influence corner separation bubble formation/suppression. A similar conclusion was made over two decades ago by Chen, ${ }^{18}$ regarding secondmoment Reynolds-stress closures' advantages for this type of flow.

Experimentally, Simpson and co-authors have investigated juncture flows in great detail (see, e.g., Refs. 19-22). However, that body of work did not include much focus on trailing-edge corner separation. Instead, the focus was more on the leading edge region and the horseshoe vortex behavior. For example, they found that there is a lowfrequency unsteadiness of the instantaneous flow structure associated with the horseshoe vortex, as evidenced by bimodal histograms of velocity fluctuations. This bi-modal behavior has since been computed with a hybrid combination of Reynolds-averaged Navier-Stokes and Large Eddy Simulation (hybrid RANS-LES). ${ }^{23}$

More recently, ONERA has conducted a series of important separated juncture flow investigations, involving both experiment and CFD. Gand et al. ${ }^{7,10,24}$ described a simplified wing-body juncture experiment that used a NACA 0012 wing mounted to a flat plate. Although designed to achieve a corner separation (based on the model's low "bluntness factor"), the flow in the wind tunnel experiment remained attached. In the numerical investigation, all RANS models employed—including a Reynolds stress model—produced corner separation, contrary to the experiment. However, a LES calculation agreed well with the experimental data in this region (with the exception that the convection of the horseshoe vortex was "not completely satisfactory"). Subsequently, the investigators re-designed the experiment with a twisted NACA 0015 wing on a plate. ${ }^{25}$ This time, the corner flow separated in the experiment. Details were provided at a chord Reynolds number of $1.2 \times 10^{6}$ and angle of attack of $12^{\circ}$, although other conditions were also investigated. Experimental details included wall pressure spectra obtained using unsteady pressure sensors and flowfield measurements of mean and turbulent quantities from stereoscopic High-Speed Particle Image Velocimetry (HSPIV). The authors noted that the corner flow separation onset was delayed when the oncoming boundary layer was thicker. The ONERA group conducted CFD analysis of the twisted NACA 0015 configuration in Bordji et al. ${ }^{6,26}$ with RANS. Here, they noted that the SA model in conjunction with the QCR closure yielded improved predictions compared to the linear SA model, which grossly overpredicted the extent of corner separation.

As this is not a survey paper, we do not describe other past juncture flow efforts here. References to other juncture flow work can be found in Gand et al. ${ }^{7}$ and Simpson, ${ }^{19}$ among others. Given the large variations in past CFD computations of separated juncture flowfields (at the DPWs, for example), experimental efforts to characterize and quantify the juncture flow physics-like those done by the ONERA group-are extremely important. Although the last several years have seen apparent CFD improvements through the use of Spalart's QCR closure, a good understanding of juncture flow physics remains elusive, and many questions still remain. For example, if better prediction of Reynolds stresses near the corner is the primary driver for attaining appropriate separation bubble characteristics, then why did the use of a Reynolds stress closure yield poor results in Ref. 24? In other words, we now presume that nonlinear turbulence modeling is better for predicting this type of flow field, but existing comparisons are not all consistent. Furthermore, we have only very limited quantitative data with which to take the next steps to thoroughly validate (and hopefully improve) existing models. Additional definitive experiments like ONERA's are needed to help guide CFD.

The idea for the NASA Juncture Flow experiment was originally conceived by a few members of the DPW ${ }^{1}$ steering committee. The experiment is intended to be primarily for the purpose of CFD validation for wing-juncture trailing edge separation onset and progression. Unlike the ONERA experiment, which used an unswept wing mounted on a flat plate, the NASA Juncture Flow experiment plans to use a swept wing-body configuration. To eliminate questions regarding the possible influence of tunnel wall boundary layers, full-span testing will be employed. Furthermore, as will be described in Section II below, a significant amount of effort is planned for attaining flowfield details very near the walls in the corner region, and for duplicating measurements via different techniques. Guidelines delineated in Aeschliman and Oberkampf ${ }^{27}$ and Oberkampf and $\operatorname{Roy}^{28}$ for design and execution of validation experiments will be followed. Although not necessarily trustworthy, some preliminary CFD analyses have already been made for several candidate design configurations; these will be briefly outlined in Section III. Because of high uncertainties associated with designing a configuration to achieve the desired juncture flow behavior with CFD tools that may be inaccurate, a cautious approach was adopted for this study (recall the first ONERA test with the NACA 0012 wing that unexpectedly failed to yield any separation). In this cautious approach, several preliminary "risk reduction" experiments have been conducted, as described in Section IV. The risk reduction experiments allow the team to more confidently select a final wing configuration that provides the desired flowfield characteristics; they also help to determine the appropriate 
placement of instrumentation for the final experiment. This paper ends with a brief description of future plans for the NASA Juncture Flow experiment, and concluding remarks.

\section{Goals and Purpose of the Juncture Flow Experiment}

A Juncture Flow team, led by NASA, has been active for several years. The team currently consists of members from NASA Langley, NASA Ames, Boeing, and Penn State, as well as several consultants. In mid-2014, concrete plans for definitive experimental measurements started to take shape. Several watershed moments occurred early in this process. First, the team decided to make use of an internal Laser Doppler Velocimetry (LDV) system that will be mounted inside of the fuselage on a movable three-axis traverse system; it will measure the flow field very near the wing-body juncture through window(s) in the fuselage. See the schematic diagram in Fig. 1. Preliminary estimates dictated that this system will require a cross-sectional space of at least 13.7 inches high by 10.3 inches wide, with a likely need for an additional 2 inches vertically. This requirement fixed the minimum size of the juncture flow model. Complementary measurements with particle image velocimetry (PIV) are also planned.

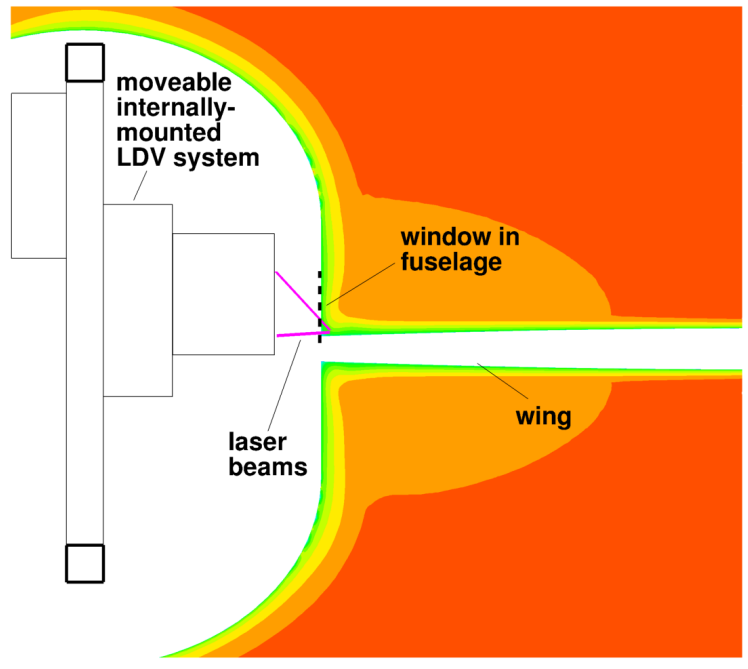

Figure 1. Schematic of internal LDV system measuring in the wing-body juncture region.

Initial efforts were focused on devising a transonic flow application, primarily because the DPWs had used freestream Mach numbers in this range. However, there were three significant difficulties with this approach: (1) the use of CFD to design a configuration that attained all of the desired characteristics proved to be challenging (the influence of the wing's shock wave on the juncture separation bubble was unclear and represented a possible complicating factor); (2) the number of available transonic wind tunnel facilities of sufficient size was somewhat limited; and (3) CFD modeling of the walls in the transonic facilities considered could introduce a high degree of uncertainty. The team initially struggled to try to overcome these obstacles. The NASA Ames 11 foot tunnel was considered for the transonic testing, but in the end the requirements for body size to comfortably fit the internal LDV system in a full-span model were too big (too much blockage) for that tunnel. Furthermore, some smaller-scale risk-reduction experiments were envisioned, and subsonic testing venues were far easier to find. So the decision was finally made to try to design the experiment at subsonic conditions.

As mentioned earlier, the primary goal for the NASA Juncture Flow experiment is to acquire CFD-validation quality data for wing-juncture trailing edge separation onset and progression on a full-span wing-body model. By "CFD-validation quality" we mean that the experiment should include the measurements of all information-including boundary conditions, geometry information, and quantification of experimental uncertainties-necessary for a thorough and unambiguous CFD validation study. ${ }^{27}$ The attainment of this data will then enable the assessment of CFD models and sub-models. The main purpose of the CFD model assessment is as follows:

Assess the ability of existing models to predict the onset and extent of the three-dimensionally separated flow near the Wing Juncture Trailing Edge (WJTE) region of a full-span wing-body configuration, in terms 
of the surface topology of the flowfield structure. To provide a range of prediction difficulty, a variation of WJTE flow fields are required, including the onset and progression of corner separation.

As will be shown in Section III, to date with CFD analysis, the team has had difficulty finding a wing-body design that achieves both fully attached flow and separated flow via a simple mechanism such as variation of angle of attack (to simplify the process of CFD validation, the geometry should remain fixed; i.e., not achieve separation by changing or adding new geometric features). However, attainment of a range of attached through separated flow remains a primary goal. Whether it is achievable in practice remains to be seen.

Other experimental goals include additional details considered desirable for CFD validation. For example, timeaveraged velocities, Reynolds stresses and triple moments, surface pressures, surface shear stress, and time-dependent quantities are desired. The following represents a prioritized list of desired data (top priority first):

1. Documentation of the test article geometry and surface, as fabricated, as assembled, and as tested in the wind tunnel.

2. Tunnel boundary conditions upstream, around, and downstream of the test article for each angle of attack condition tested.

3. Mean velocity components through the boundary layer on the fuselage and wing (both upstream and within the WJTE region).

4. Second moments (Reynolds stress tensor components) through the boundary layer (same locations as in Item $3)$.

5. Time-averaged surface pressure coefficients on the fuselage and wing.

6. If boundary layer trip(s) are used, details regarding their location, type, attachment, etc.

7. Documentation of the surface geometry of the wind tunnel test section and the contraction section upstream of the test section.

8. Documentation of the geometry of the mounting hardware and cabling for the test article in the test section.

9. Time-series measurements of angular position and acceleration on-board the test article.

10. Surface shear stresses on the test article (both upstream and within the WJTE region).

11. Spectra of unsteady velocity and pressure at various locations on the fuselage and wing (both upstream and within the WJTE region).

12. Freestream turbulence intensity and turbulence length scale.

13. Triple velocity correlations through the boundary layer (same locations as in Item 3).

The above items may or may not be possible to attain in this test; the prioritization will be used during testing to help make decisions when time is limited or when particular capabilities are not available. All of the mathematical model input data described above should include a rigorous estimation of total experimental uncertainty, i.e., both random (precision) uncertainty and systematic (bias) uncertainty.

\section{Preliminary Configurations and CFD Analysis}

CFD was used to design and analyze a variety of different wing-body configurations. Early in the development effort, a simple fuselage configuration was settled upon, with the CRM fuselage configuration as a starting point. However, this new Juncture Flow Model fuselage was modified to include two planes of symmetry (top-bottom and left-right), and its sides are vertical and flat in the vicinity of the wings, for greater ease in including windows for the internally-mounted LDV laser beams to pass through. The fuselage includes a nose, and constricts down in the rear region toward where the sting is attached. Fig. 2 shows a view of the fuselage shape. Although not decided yet, the fuselage design for the final model may include some provision for varying its length upstream of the wing.

For the wings, several different designs were explored with CFD, by using a realistic-looking planform shape that was truncated in the spanwise direction in order to allow for an overall larger model. For all of the wings, 


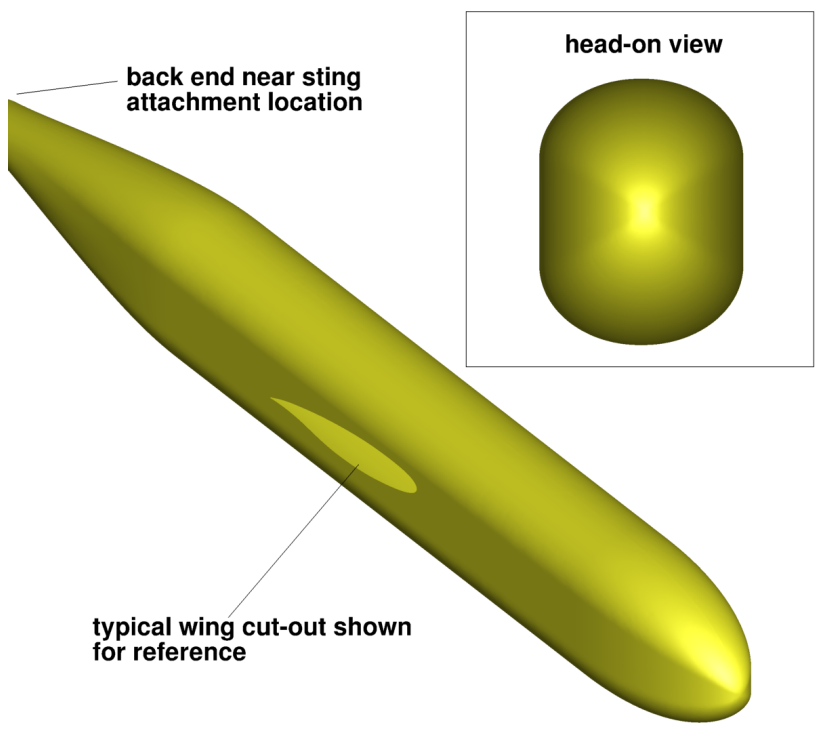

Figure 2. Schematic of the currently-defined Juncture Flow Model fuselage shape.

predicted trailing-edge juncture separation bubble sizes were noted as a function of angle of attack. The goal was to find design(s) for which there would be attached flow at low angles of attack and a quickly-growing bubble at higher angles of attack. Optimally, a symmetric wing is desired, because one could then take advantage of the top-bottom symmetry to exploit design-of-experiment ideas for attaining uncertainty information. However, the team also made use of a truncated version of the wing from the DLR-F6 configuration (which is not symmetric). In past transonic experiments the DLR-F6 wing-body had yielded corner separation bubbles, so the team believed that this wing had a high likelihood of achieving corner separation in spite of differences in the free stream, fuselage shape, and wing span.

In the CFD, the SA-RC turbulence model, ${ }^{29}$ combined with the QCR closure ${ }^{14}$ was primarily used. The reason for this choice was undocumented positive experiences among team members (also see the Introduction for a discussion of the published success of SA-QCR for corner flows). Other models were also tried. For example, the seven equation Reynolds stress model SSG/LRR-RSM-w $2012^{30}$ was found to yield similar qualitative results to SA-RC-QCR overall.

In addition to the truncated DLR-F6 wing (hereafter referred to as simply "F6"), the team ended up with four other trial wings. Unlike the F6, which is non-symmetric and also includes wing dihedral, these others were all top-bottom symmetric. All wings had somewhat similar planform shapes, including a "Yehudi break," or crank, part-way out the span, although the F6 wing had slightly different leading- and trailing-edge sweep angles, crank location, and crank chord than the others. One wing, referred to as "0015," used a NACA 0015 root shape. This was blended to a NACA 0012 at the crank, and NACA 0010 at the tip. The reason for the blending was to reduce the wing thickness and hence the likelihood of outboard trailing-edge separation. A second wing, referred to as "0015mod," used a modified NACA 0015 root shape, for which the last 2 coefficients in the airfoil equation were altered to achieve greater adverse pressure gradient over the rear of the wing, while maintaining the same trailing edge thickness. This wing was blended into the same NACA 0012 and NACA 0010 as the "0015" wing. A third wing, referred to as "F6-S12," was designed based on a symmetrized version of the F6 wing, with increased maximum thickness and trailing edge included angle in order to achieve significant adverse pressure gradient over the rear part of the wing, to encourage the likelihood of trailing edge separation at the root. Rather than blend to a NACA 0012 and 0010, the F6-S12 blended to symmetric airfoil shapes derived from the original CRM design. The fourth wing, referred to as "COCA," was designed with CDISC, ${ }^{31}$ with the constraint of achieving a specified skin friction distribution at two stations near the root (for example, $C_{f}=0.0001$ at the root trailing edge at zero degrees angle of attack, with specified $d\left(C_{f}\right) / d x$ upstream). A curvature constraint ahead of the wing's maximum thickness was also employed. This wing blended to the crank and tip in the same fashion as the F6-S12.

Leading edge fillets or "horns" (in the leading edge corner region between the wing and fuselage) were also created for each wing shape. One purpose of the horn is to help eliminate and/or mitigate the development and influence of the horseshoe vortex. A discussion of the effect of horns can be found in Devenport et al. ${ }^{21}$ Note that the horns used here were geometrical fairings only, and were not aerodynamically designed/optimized. They were created by fixing locations on the fuselage and wing, and fairing a smooth leading-edge curved shape between them (tangent at the wing 
and approximately $13^{\circ}$ at the fuselage), while maintaining continuity everywhere with the wing slope. The team has not yet decided whether or not to include a horn on the final configuration. The various wing root shapes are shown in Fig. 3 (without horns). Here, the F6 is clearly seen as the only non-symmetric wing shape, and the F6-S12 and the COCA are by far the thickest wing shapes. Two of the complete wing-body configurations (the F6 with horn and 0015mod with horn) are shown in Fig. 4. The other wings (0015, F6-S12, and COCA) generally look similar in their planform shapes to the $0015 \mathrm{mod}$ in Fig. 4(b).

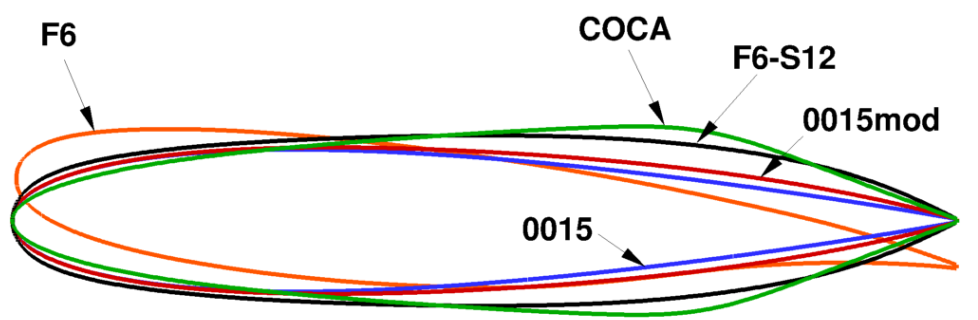

Figure 3. Wing shapes at the root (horns off).

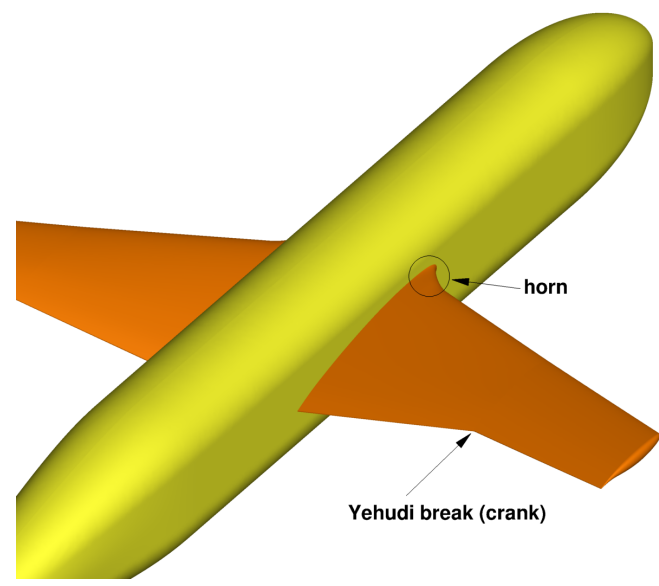

(a) F6 with horn

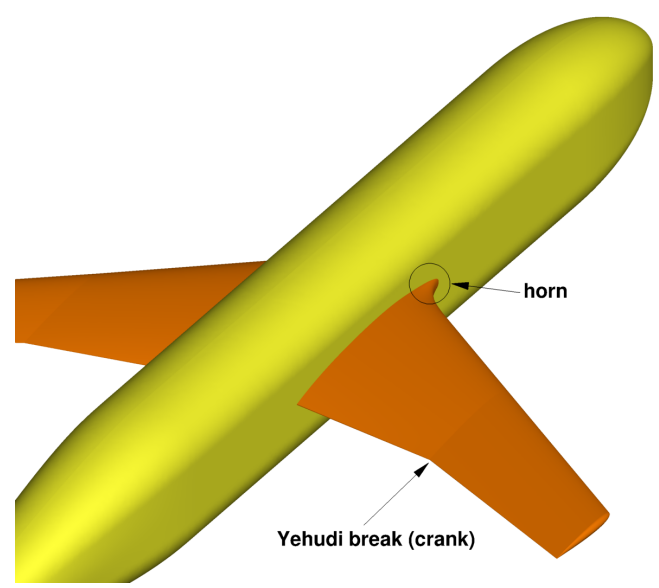

(b) $0015 \mathrm{mod}$ with horn

Figure 4. Views of two of the wing-body configurations.

Figure 5 shows plots of approximate computed bubble size (length and width) as a function of angle of attack for the various wing-body configurations. No error bars are included in the plots, but the uncertainty in the computations is considered to be quite high. Computations have been made both with OVERFLOW ${ }^{32}$ and FUN3D, ${ }^{33}$ which have proved to be reasonably consistent with each other when using SA-RC-QCR. Here, only FUN3D results are shown, for $M=0.2$ and $R e_{c}=3.5$ million based on $c=$ crank chord (the particular separation bubble sizes are given in inches, for a configuration with crank chord of around 22 to 23 inches). The crank chord was chosen for length scale because it is an easily visible reference on the models, which is helpful for this project because the team is dealing with many differently-sized scale versions of the configurations. Lower Reynolds numbers have also been run, yielding a trend of somewhat larger bubbles. Note that the details are not important here, only whether or not a bubble is predicted, and the sizes of the bubbles relative to each other. Based on previous studies, the team believed that the CFD probably had a tendency to predict too large a bubble compared to experiment. So the goal was to achieve an array of different results, leaning toward larger bubble sizes. The F6 wing produced the largest bubble length and the 0015 produced the smallest, while the 0015mod, COCA, and F6-S12 each fell somewhere in between. Bubble width tended to be more significant for the F6-S12 and COCA configurations. Although not detailed here, the effect of horn on versus horn off was relatively minor, with the former generally producing slightly larger separation bubbles. Figure 6 shows example 


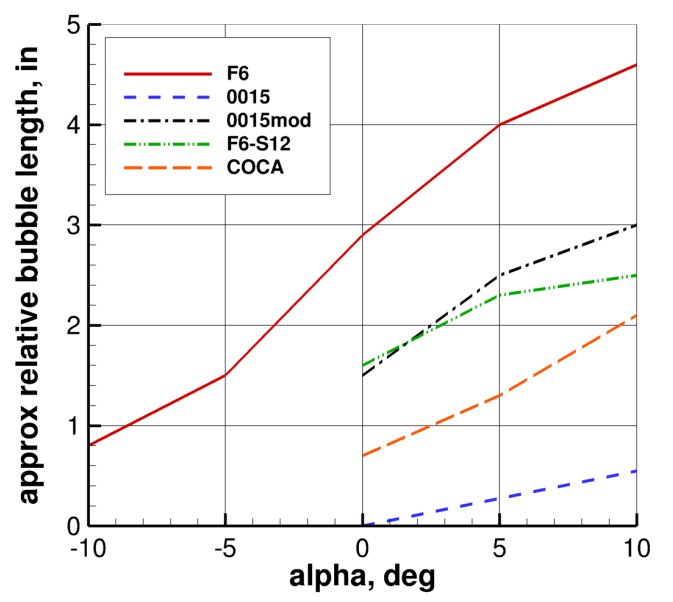

(a) Bubble length

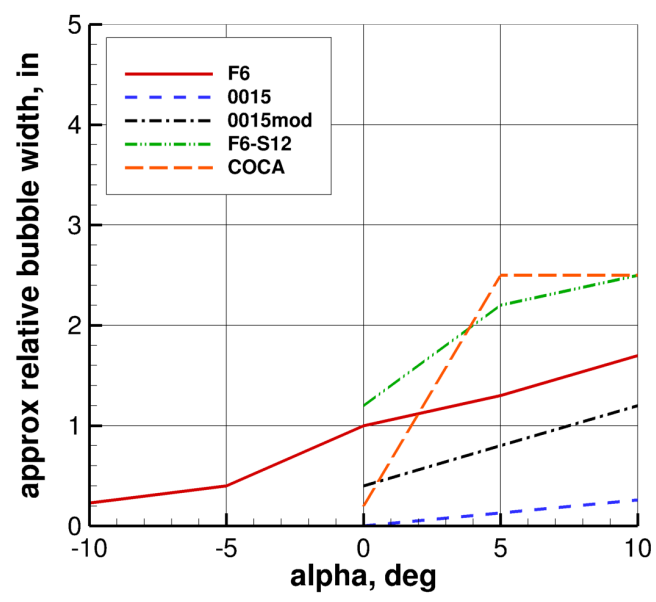

(b) Bubble width

Figure 5. Approximate relative bubble sizes predicted by CFD for the various wings (with FUN3D).

CFD-generated streamlines from the five wing designs, in this case at angle of attack of 5 degrees and $R e_{c}=3.5$ million. The F6 and the 0015 mod each yielded "traditional-shaped" bubbles (i.e., what would normally be expected on typical wing-body configurations), and the $0015 \mathrm{mod}$ also produced some trailing edge separation slightly outboard of the bubble. The 0015 yielded an extremely tiny bubble at this angle of attack. The F6-S12 and COCA designs both yielded oddly-shaped bubbles with a tendency toward larger bubble widths than the F6 and 0015mod.

This set of five wing configurations was felt to encompass a reasonably broad array of possible outcomes in terms of juncture bubble behavior. A few other wing configurations beside these five were also tested, but they either did not yield the desired behavior or did not add any value beyond what the other five wings provided; so they are not described here. Because of low confidence in the CFD predictions of corner separation size, the team decided to test all five wings in a series of preliminary "risk-reduction" wind tunnel tests. These tests are described next.

\section{Risk Reduction Wind Tunnel Tests}

In this section, an overview of the risk reduction wind tunnel tests is provided, followed by some sample results from one of the tests.

\section{A. Overview}

For the final wind tunnel test, the model size is planned to be at $8 \%$ scale (as compared to a representative full-scale CRM-like aircraft configuration). At this scale, the crank chord of the F6 wing will be approximately 22 inches, the total wing span will be approximately 131 inches (tip to tip), and the fuselage outer mold line will be approximately 24.8 inches high by 18.6 inches wide, large enough to easily fit the planned LDV system as well as any needed cabling. The approximate length of the fuselage will be somewhere in the neighborhood of 200 inches (depending on where the design shape gets terminated to fit to a sting mount). The Reynolds number based on crank chord at $M=0.2$ is expected to be approximately $2.3-2.7$ million.

However, preliminary risk-reduction wind-tunnel tests were conducted first (in mid- to late-2015), in order to help the team determine the final wing configuration. Table 1 summarizes all of the experiments. One of the risk-reduction experiments (labeled "Pre 1" in the table) was a limited semi-span low Reynolds number test in the TC2 facility at NASA Ames. This facility has a test section size of 48 inches by 32 inches and operates subsonically. It was chosen because of its low cost and relatively rapid turnaround. However, use of a semi-span model was considered to be risky (potentially unreliable) due to influences of the wall boundary layer and additional wall-fuselage juncture flow on the wing-fuselage juncture flow of interest, so only two wing shapes were built. The models built for this test were at $3 \%$ scale. At this scale, the crank chord of the F6 wing was about 8.2 inches, wing semi-span was about 24.6 inches, and Reynolds number was approximately 600,000 - 700,000. Limited testing was done for the F6 and F6-S12 wings, and 
both surface oil flow photographs and oil film interferometry data were acquired.

The second wind tunnel experiment ("Pre 2" in the table) was a full-span low Reynolds number test in the Virginia Tech Stability Wind Tunnel. This tunnel has a 6 foot by 6 foot test section and operates subsonically. The models built for this test were at $2.5 \%$ scale. At this scale, the crank chord of the F6 wing was about 6.9 inches, wing tip-to-tip span

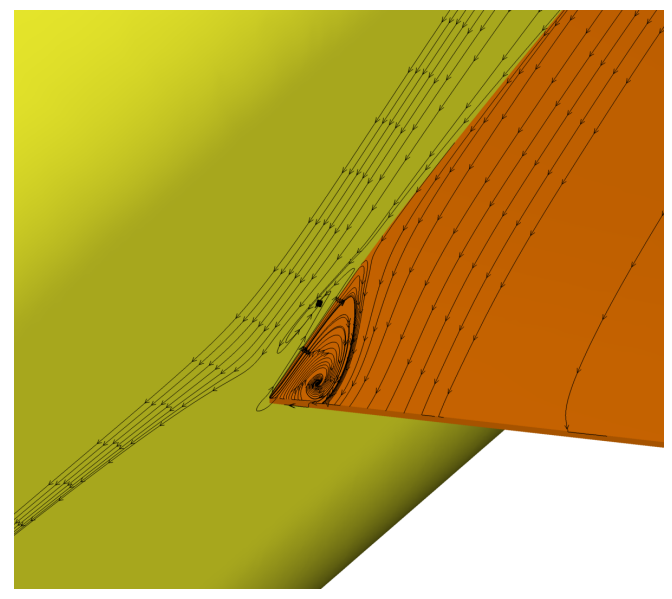

(a) F6

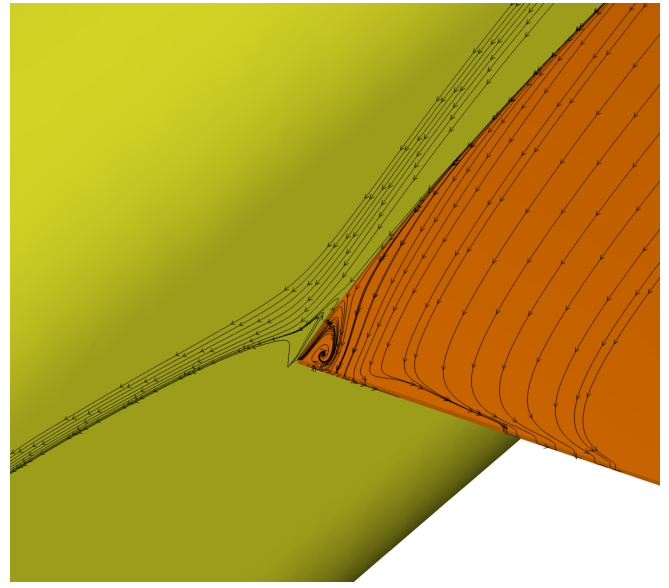

(c) $0015 \mathrm{mod}$

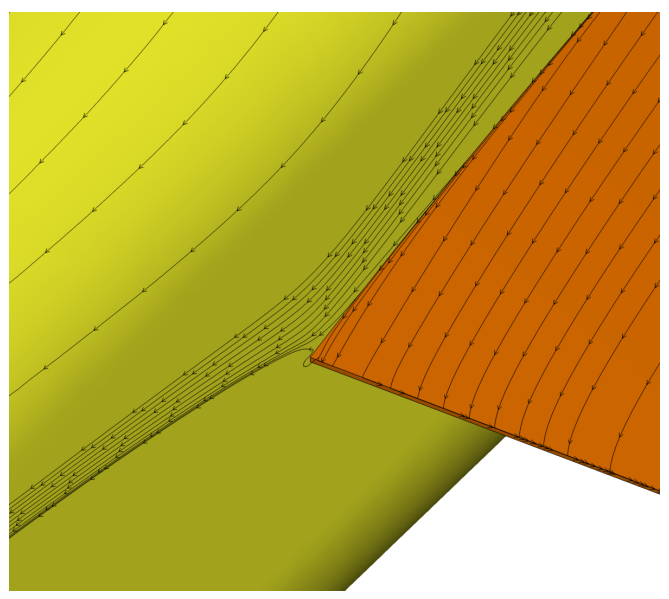

(b) 0015

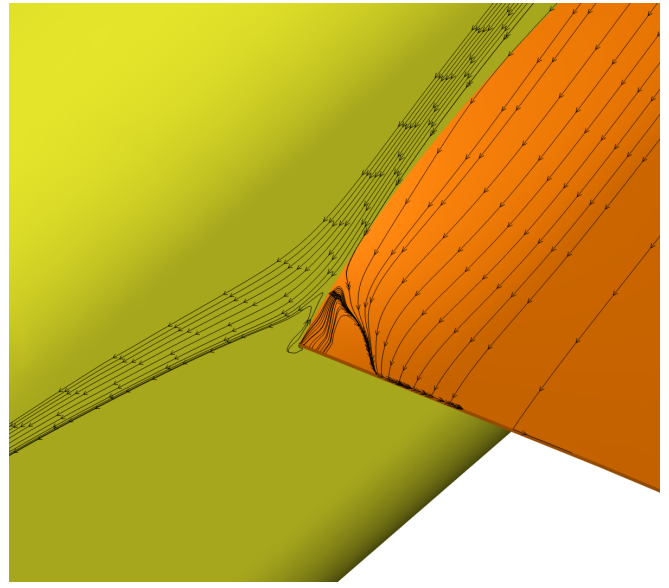

(d) F6-S12

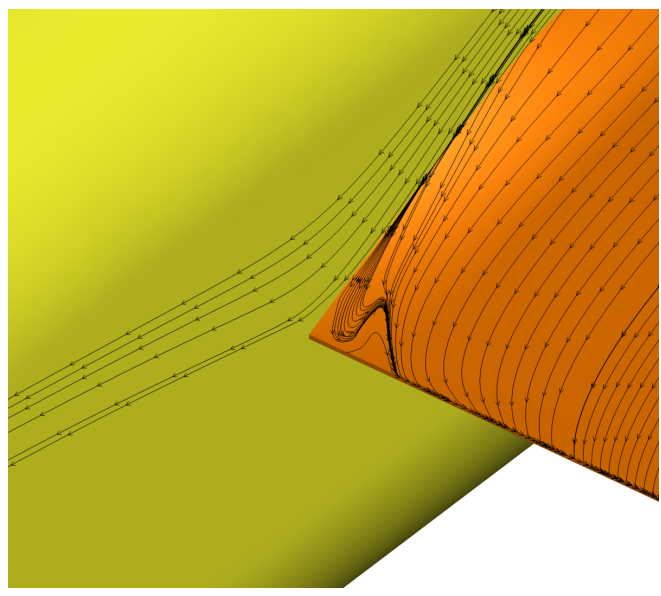

(e) $\mathrm{COCA}$

Figure 6. Example streamlines from CFD for the five configurations (with FUN3D at $\alpha=5^{\circ}, M=0.2$, and $R e_{c}=3.5$ million). 
Table 1. Summary of wind tunnel tests (using F6 wing for reference)

\begin{tabular}{llllllll} 
Test & Tunnel & Date & Model size & $R e_{c}\left(\times 10^{6}\right)$ & $M$ & Crank chord, $c$ (in) & Wing span (in) \\
\hline Pre 1 & TC2 & Summer 2015 & $3 \%$ & $0.6-0.7$ & 0.14 & 8.2 & 24.6 (semi-span) \\
Pre 2 & VA Tech & Summer 2015 & $2.5 \%$ & $0.6-0.7$ & 0.18 & 6.9 & 40.9 \\
Pre 3 & $14 \times 22$ & Fall/Winter 2015 & $6 \%$ & $2.3-2.7$ & 0.27 & 16.5 & 98.3 \\
Final & $14 \times 22$ & TBD & $8 \%$ & $2.3-2.7$ & 0.20 & 22.0 & 131.0
\end{tabular}

was 40.9 inches, and Reynolds number was similar to that for the TC2 test, approximately $600,000-700,000$. All five wing shapes (F6, 0015, 0015mod, F6-S12, and COCA) were tested in this tunnel. The wings were interchangeable onto the fuselage, and the wings could also be tested with and without a horn. To minimize the model construction costs, six unique wings were built: F6 (port), F6 (starboard), 0015 (port), 0015mod (starboard), F6-S12 (port), and COCA (starboard). The following wing pairs were always tested together: F6 with F6, 0015 with 0015mod, and F6-S12 with COCA. CFD analysis suggested that there would be negligible influence on separation bubble behavior from the use of slightly different wings on the port and starboard side.

Because of its low Reynolds number, this "Pre 2" test may not be representative of the results that will be ultimately obtained at the higher Reynolds number of roughly $2.3-2.7$ million. Therefore, the results from this test were to be viewed only qualitatively. The transition characteristics, with and without trip dots on the fuselage and wing upper surface, were assessed with infrared (IR) thermography. Oil flow visualizations were used to identify the trailing-edge corner separation bubble, to visualize the general flow pattern on the wing upper surface planform, and to visualize the flow pattern on the fuselage in the vicinity of the wing-fuselage juncture. This test was an invaluable precursor to the $14 \times 22$ tunnel risk-reduction test, giving the experimentalist team members experience with IR thermography and providing preliminary results (albeit at low Reynolds number). A photograph of the model in the Virginia Tech tunnel is shown in Fig. 7. Trip dots can be seen around the fuselage nose as well as near the wing leading edges. Examples of IR thermography images are given in Fig. 8. The darker shade indicates laminar flow, while the lighter shade indicates turbulent flow. Here, the untripped F6 wing is shown to be dominated by significant regions of laminar flow (especially outboard) at $\alpha=0^{\circ}$, but natural transition takes place (due to turbulent reattachment of a laminar separation bubble near the wing leading edge) somewhere below $\alpha=5^{\circ}$. Although the quality of the photographs in Fig. 8(c) and (d) is somewhat poor, they confirm that the application of the trip dots (at roughly $4 \%$ chord) successfully induced transition to turbulence.

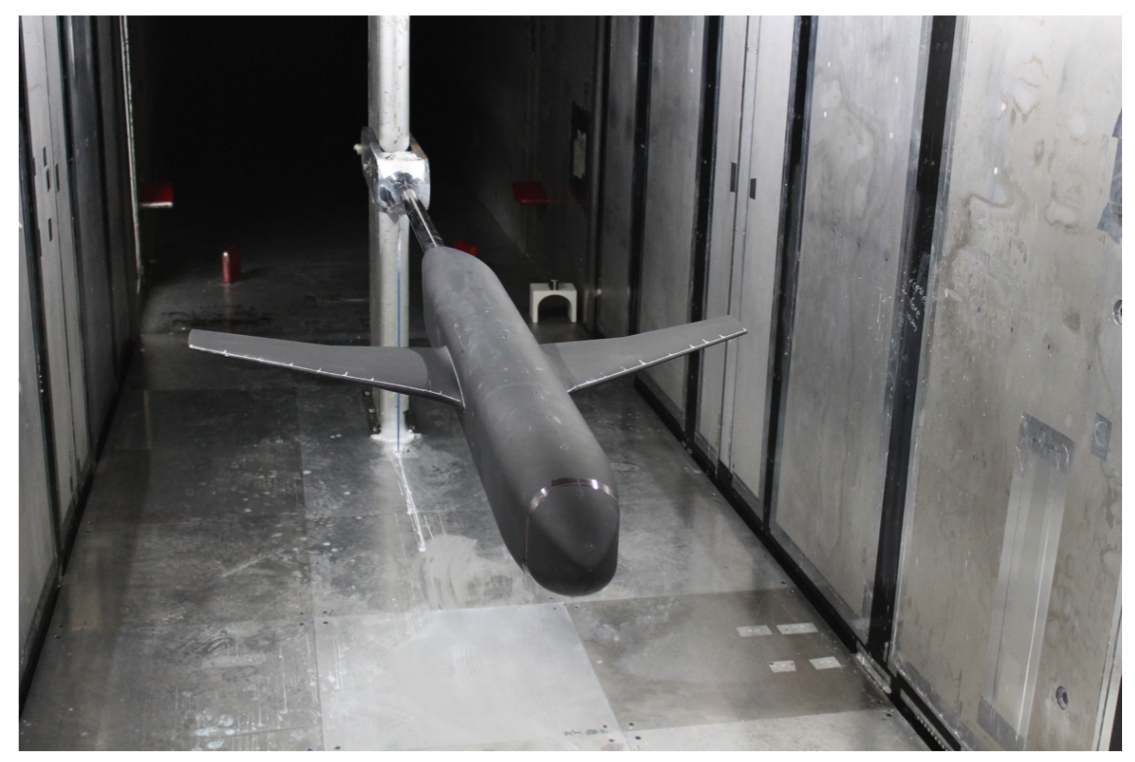

Figure 7. Photograph of the $2.5 \%$ scale model in the Virginia Tech Stability Wind Tunnel. 


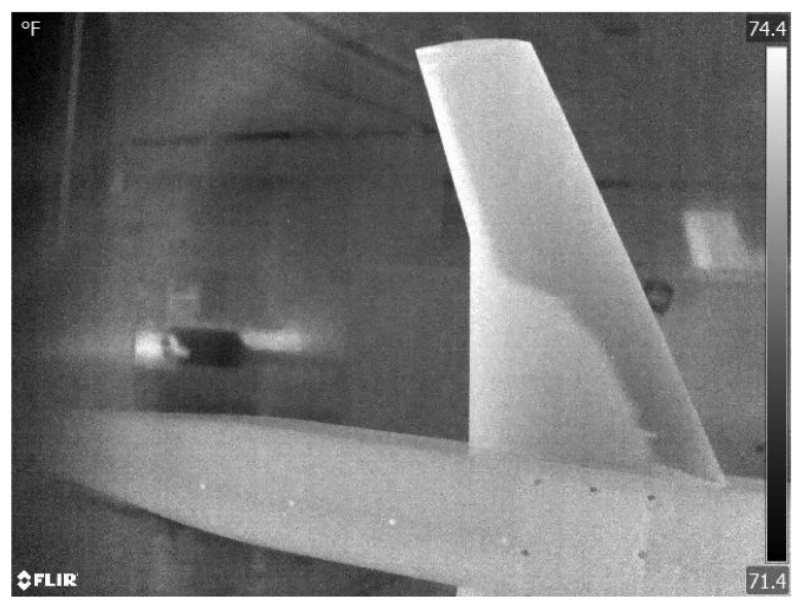

(a) Untripped, $\alpha=0^{\circ}$

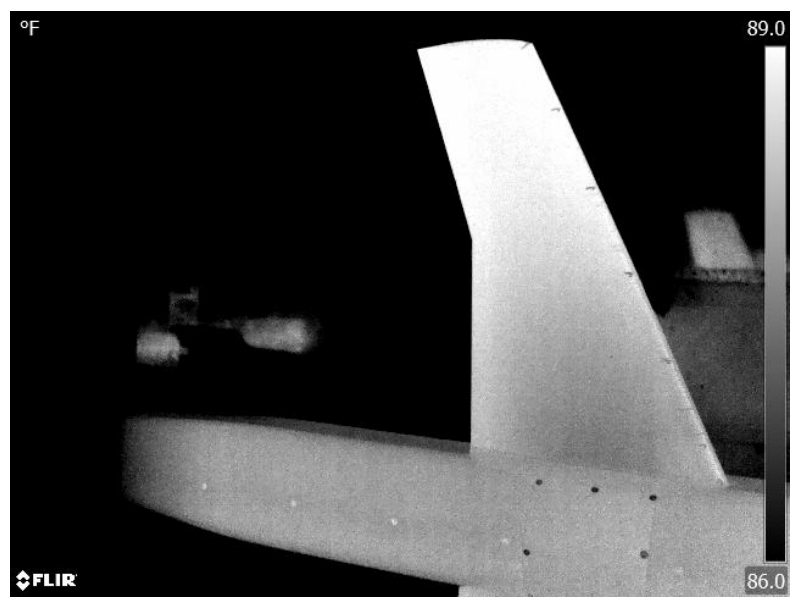

(c) Tripped, $\alpha=0^{\circ}$

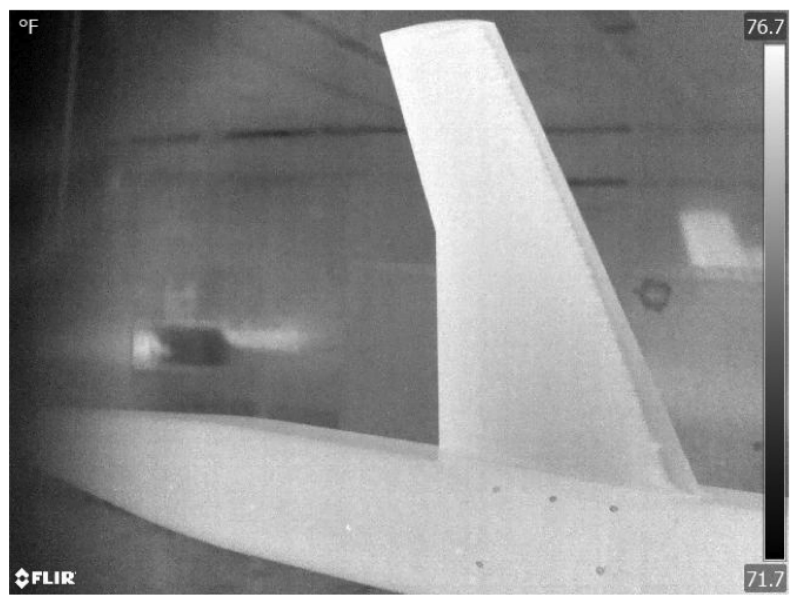

(b) Untripped, $\alpha=5^{\circ}$

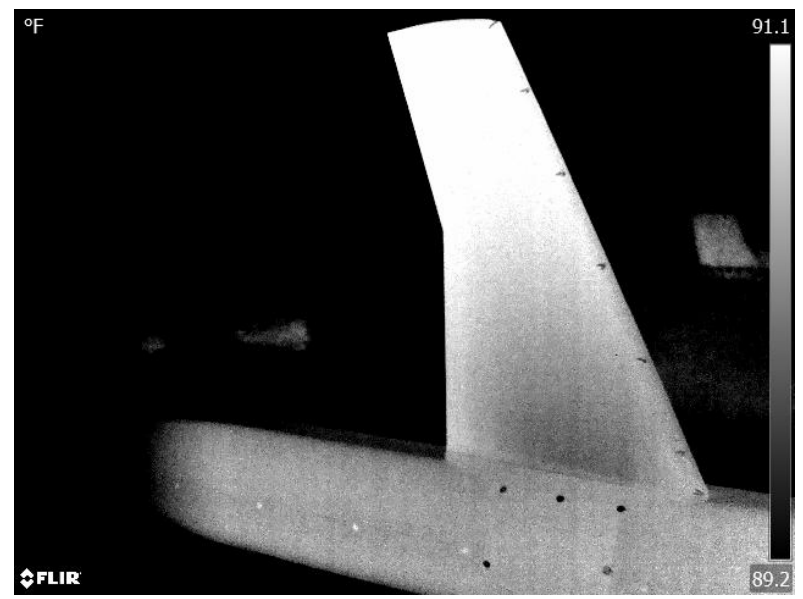

(d) Tripped, $\alpha=5^{\circ}$

Figure 8. Example IR thermography images, for the F6 wing upper surface in the Virginia Tech tunnel (flow is right to left).

The third risk-reduction wind tunnel experiment ("Pre 3" in the table) was a full-span higher Reynolds number test in the NASA Langley 14x22 subsonic wind tunnel. This tunnel has a 14 foot high by 22 foot wide test section. The models built for this risk-reduction test were at 6\% scale. At this scale, the crank chord of the F6 wing was about 16.5 inches, wing tip-to-tip span was 98.3 inches, and Reynolds number was expected to be approximately $2.3-2.7$ million. As of this writing, the $14 \times 22$ risk reduction testing has not yet taken place. All five wing shapes are expected to be tested. In this model, all wings were built with a horn, with the exception of one of the F6 wings, which can be tested either with or without a horn. Because this risk-reduction test is going to achieve similar Reynolds numbers as the final planned test, approximate bubble size information from oil-flow visualizations on the $6 \%$ scale model will be used to help the team make a decision about the final wing configuration.

\section{B. Results}

A few sample results from the Virginia Tech full-span experiment are shown in this section. Semi-span results from the TC2 tunnel turned out to be qualitatively different from the full-span tests, yielding significantly smaller separation bubbles. Therefore, the TC2 results are not shown here, since they are considered non-representative of a full-span model and will not be used to make the final wing design decision.

Figure 9 shows a sampling of oil flow photographs at $\alpha=5^{\circ}$. Here, the fuselage and wings were tripped, and the horns were included. At this angle of attack, all configurations except for the 0015 produced corner separation bubbles. These results can be compared qualitatively with the CFD predictions in Fig. 6. Although at different Reynolds numbers, there are many similarities in shape and relative behavior. Other CFD results (not shown) at the 
same low Reynolds number as the experiment (albeit run fully turbulently) yielded even better qualitative comparisons. However, we do not encourage direct comparisons between CFD and experiment here, because the risk reduction tests were not intended for validation. As mentioned earlier, the Virginia Tech test was at low Reynolds number, and therefore use of fully-turbulent CFD may not be an appropriate comparison. Also, while oil flow photographs produce useful qualitative views of what is going on, they may be unreliable for more quantitative measures. ${ }^{34}$

In terms of the behaviors of the separation bubbles with angle of attack in the experiment, all wings produced a general trend of increasing bubble size as angle of attack increased. Note that when the bubble was very small, it was impossible to tell from oil flow whether the flow was fully attached or slightly separated, because a small accumulation of fluid near the corner could indicate either state. For the F6, the bubble reached this "essentially attached" bubble size near and below approximately $\alpha=-7.5^{\circ}$. For the 0015mod, F6-S12, and COCA configurations, a bubble existed at all angles of attack (negative angles were not considered for the symmetric configurations). For the 0015, the corner flow was in the "essentially attached" state at least up through $\alpha=7.5^{\circ}$.

The oil flow was also used to explore the flow behavior on the wing all the way out to the tip, on the upper surface. An example is shown in Fig. 10 for the F6 wings at $\alpha=5^{\circ}$. In this configuration, the port side had no horn while the starboard side included a horn. With the exception of the juncture bubble and a small region of trailing-edge separation about a quarter of the way toward the crank, the upper surface flow appeared to be fully attached at this angle of attack. Overall, the effect of horn on versus horn off was relatively minor compared to the effect caused by different configurations. The oil flow can also help to provide a sense of the position and size of a horseshoe vortex, if present. Figure 11 provides an example for the F6 wing, showing the separation line on the side of the fuselage ahead of the horseshoe vortex. Knowledge of the likely location(s) of such a structure could help with the positioning of unsteady flow sensors in future tests.

\section{Future Plans}

After the successful completion of the risk-reduction test in the 14x22 wind tunnel, the team will make a determination of the best wing design for use in the final validation experiment. Then, depending on funding availability, the model will be designed and built toward the goals of collecting as much of the information prioritized earlier in Section II as possible. This design/build process could take about a year to complete. At this time, the validation experiments are planned to be conducted in the $14 \times 22$ tunnel.

\section{Concluding Remarks}

The NASA Juncture Flow Experiment has been undertaken because of a need to discover flowfield details relevant to the formation of juncture-flow separation bubbles that can occur on aerospace vehicles. CFD is currently considered unreliable for predicting such flows, so a high-quality validation experiment could go a long way toward (1) improving our understanding of the flow physics, and (2) helping CFD turbulence modelers improve their models for predicting these features. In order to mitigate the risk associated with using CFD to design the experiment, several risk-reduction experiments were conducted with five different wing designs. These wing designs were chosen to include a broad range of possible outcomes, in terms of separation bubble behavior. The results of the risk-reduction tests have been (and will be) indicators of the "true" behavior of the configurations in different tunnels and at different Reynolds numbers. One of the tests used a semi-span model, which resulted in notably different juncture flow behavior than a second full-span test at Virginia Tech. Both of these tests were at similar low Reynolds numbers (well less than a million). At the time of this writing, the most relevant risk-reduction test- to be conducted at Reynolds numbers close to that envisioned for the final validation test (near $R e_{c}=2.3-2.7$ million) - has not taken place yet. When it is completed, the NASA Juncture Flow team will select a final configuration. The final validation tests should provide publiclyavailable high-quality surface and flowfield data with unambiguous boundary conditions and geometry specifications, along with uncertainty quantification information. This future data set may then be appropriate for use in workshop environments. Ultimately, the NASA Juncture Flow test should prove to be a valuable resource for the CFD community as its practitioners continue to collectively improve and validate their predictive capabilities.

\section{Acknowledgments}

The authors would like to thank the other active members of the NASA Juncture Flow team. From NASA Langley: P. Balakumar, Mark Cagle, Dick Campbell, Jan Carlson, Judi Hannon, Luther Jenkins, Bil Kleb, Mujeeb Malik, Cathy 
McGinley, Joe Morrison, and Sandy Webb. From NASA Ames: Henry Lee, Tom Pulliam, Nettie Roozeboom, Laura Simurda, and Greg Zilliac. From Boeing: Peter Hartwich, Tony Sclafani, and John Vassberg. From Penn State: Jim Coder. From AUR, Inc.: Gwibo Byun and Roger Simpson. From WLO Consulting: Bill Oberkampf. The entire team is thanked for their dedication and perseverance.

Team members Jim Coder and Tony Sclafani deserve credit for their work in creating the F6-S12 wing; similarly Dick Campbell and Jim Coder deserve credit for the creation of the COCA wing. Team members Joe Morrison (the instigator and previous leader of this team) and John Vassberg are two of the founding members of the DPW steering committee who have helped to push the NASA Juncture Flow Experiment from idea toward reality. Additionally, among other valuable insights, John Vassberg came up with the idea of manipulating the NACA 0015 equation to encourage more trailing edge separation, as well as the idea of blending to different airfoil shapes out the span. Team member Henry Lee is thanked for going beyond the call of duty in his exceptional CFD work with OVERFLOW.

The authors gratefully acknowledge the Virginia Tech tunnel staff for their outstanding work during the $2.5 \%$ scale risk-reduction test: Aurelien Borgoltz, Matt Kuester, Tim Meyers, Bill Oetjens, and John Spidi. And thanks also go to William Devenport, Todd Lowe, and Eric Paterson for their hospitality and helpful discussions.

The authors give special acknowledgment to Scott Brynildsen of NASA's GEOLAB, for his impressive skills in developing and manipulating the geometry files for all of the Juncture Flow configurations. The authors also acknowledge Fabien Gand of ONERA for his valuable insights during discussions held when he visited NASA Langley in mid-2015.

This project has benefited from funding through NASA's Transformational Tools and Technologies (TTT) project in the Transformative Aeronautics Concepts Program (TACP). 


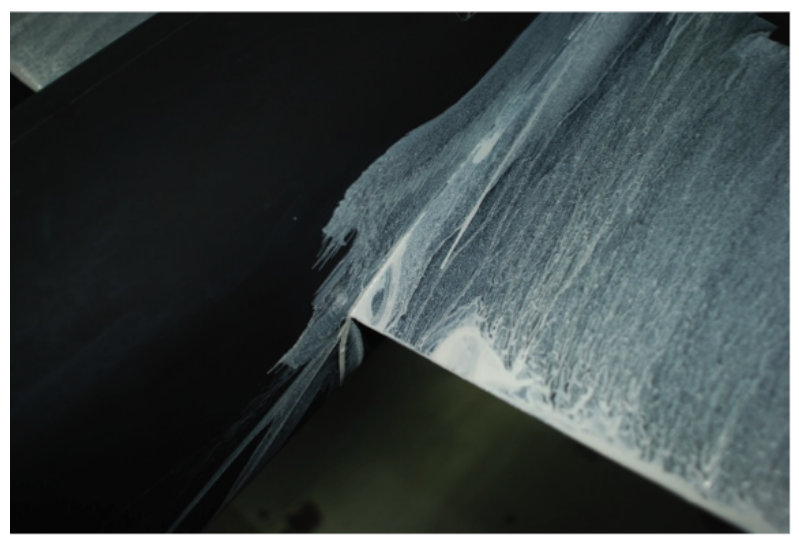

(a) F6, starboard (flow is top right to bottom left)

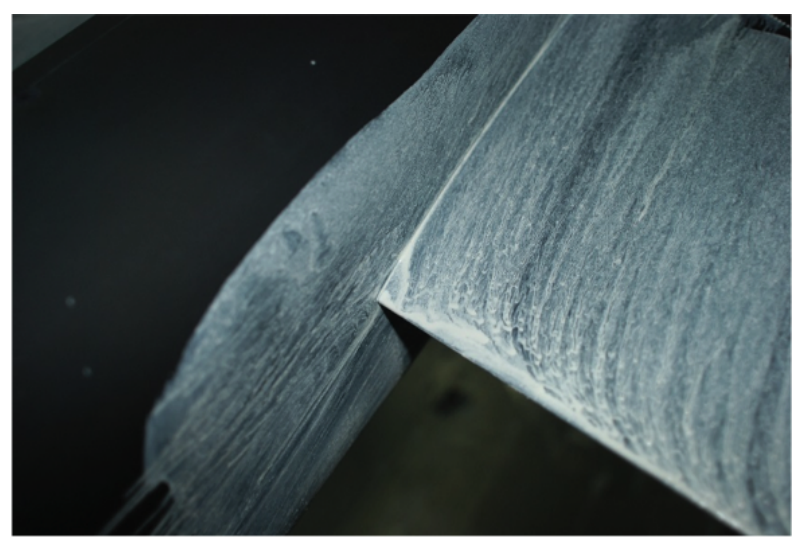

(c) $0015 \mathrm{mod}$, starboard (flow is top right to bottom left)

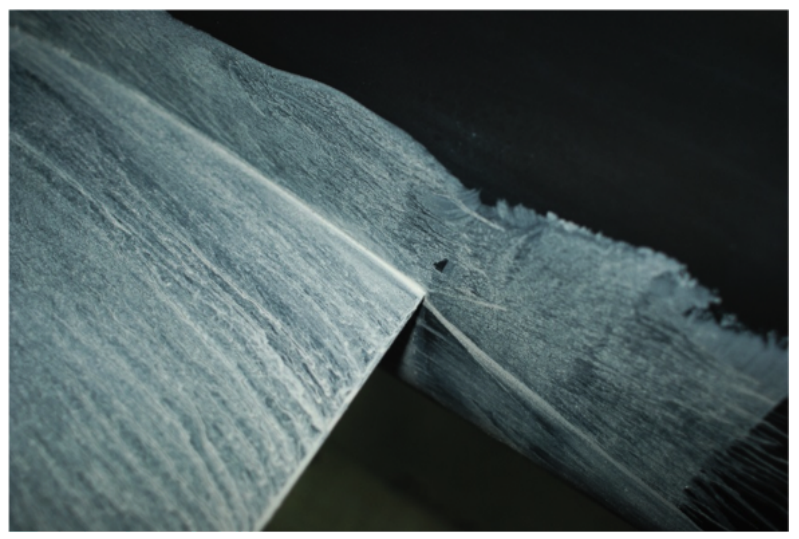

(b) 0015 , port (flow is top left to bottom right)

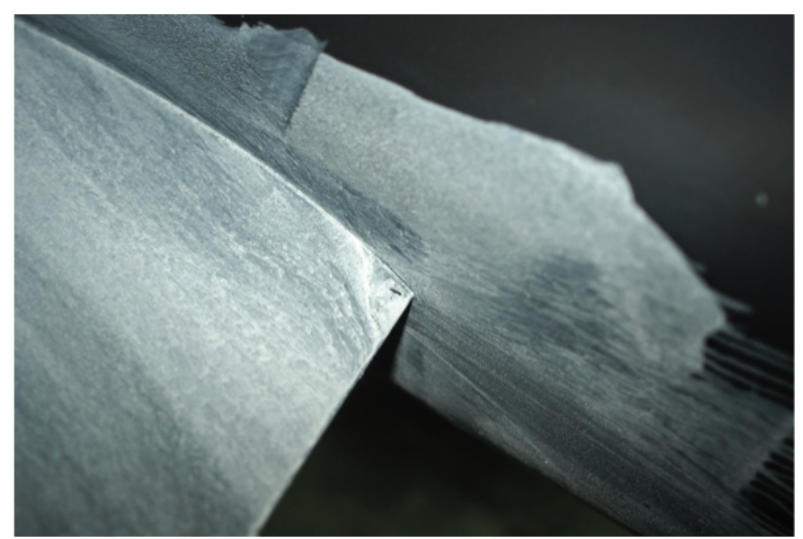

(d) F6-S12, port (flow is top left to bottom right)

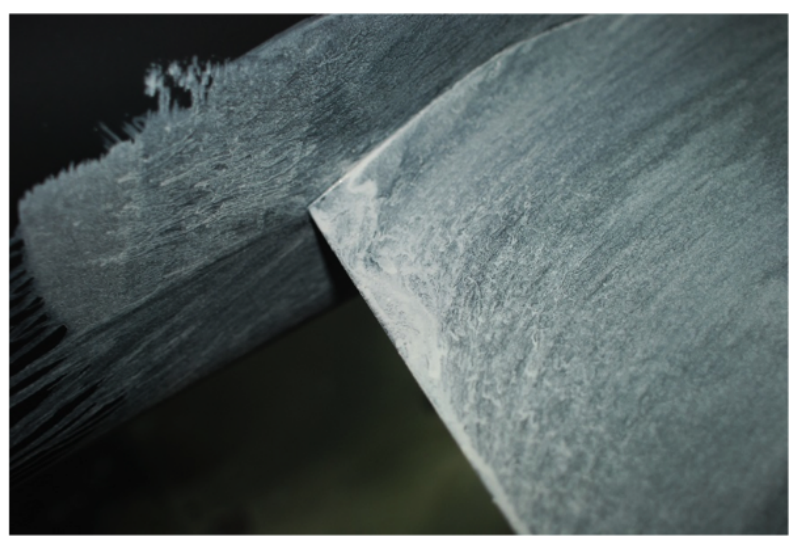

(e) COCA, starboard (flow is top right to bottom left)

Figure 9. Example oil flow photographs from the low-Reynolds-number Virginia Tech risk-reduction test $\left(\alpha=5^{\circ}\right)$. 


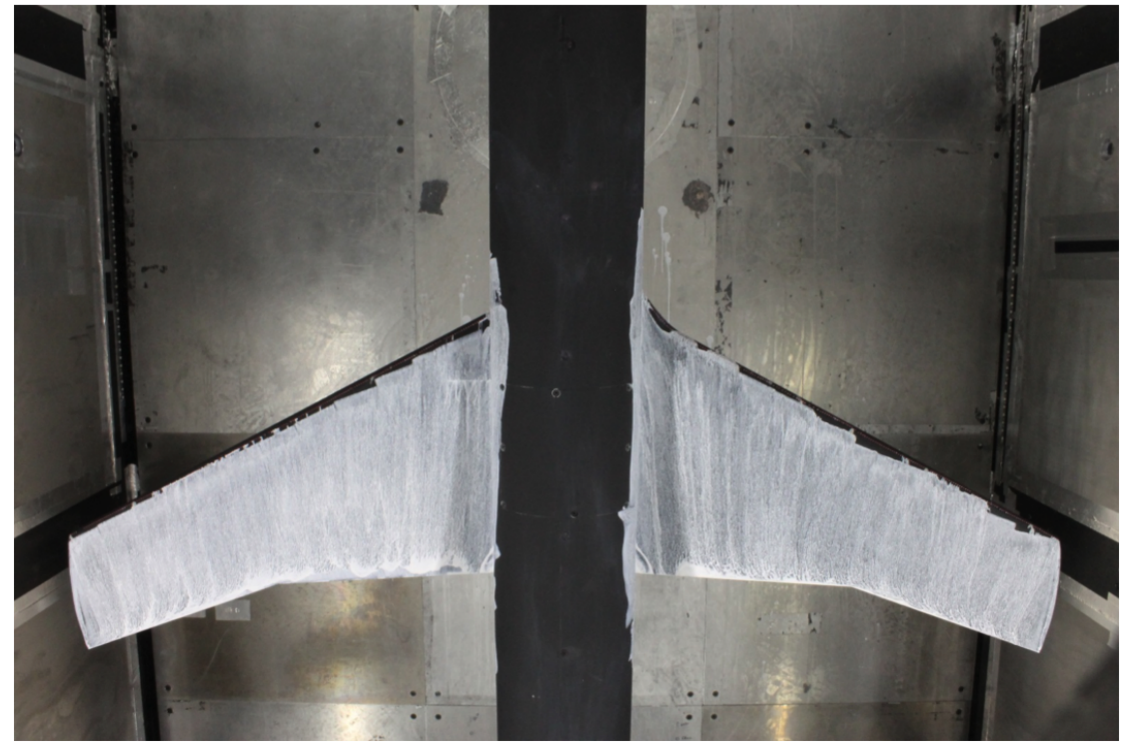

Figure 10. Photograph showing oil flow over both wings (F6 with no horn on port side, F6 with horn on starboard side), $\alpha=5^{\circ}$ in the Virginia Tech tunnel (flow is top to bottom).

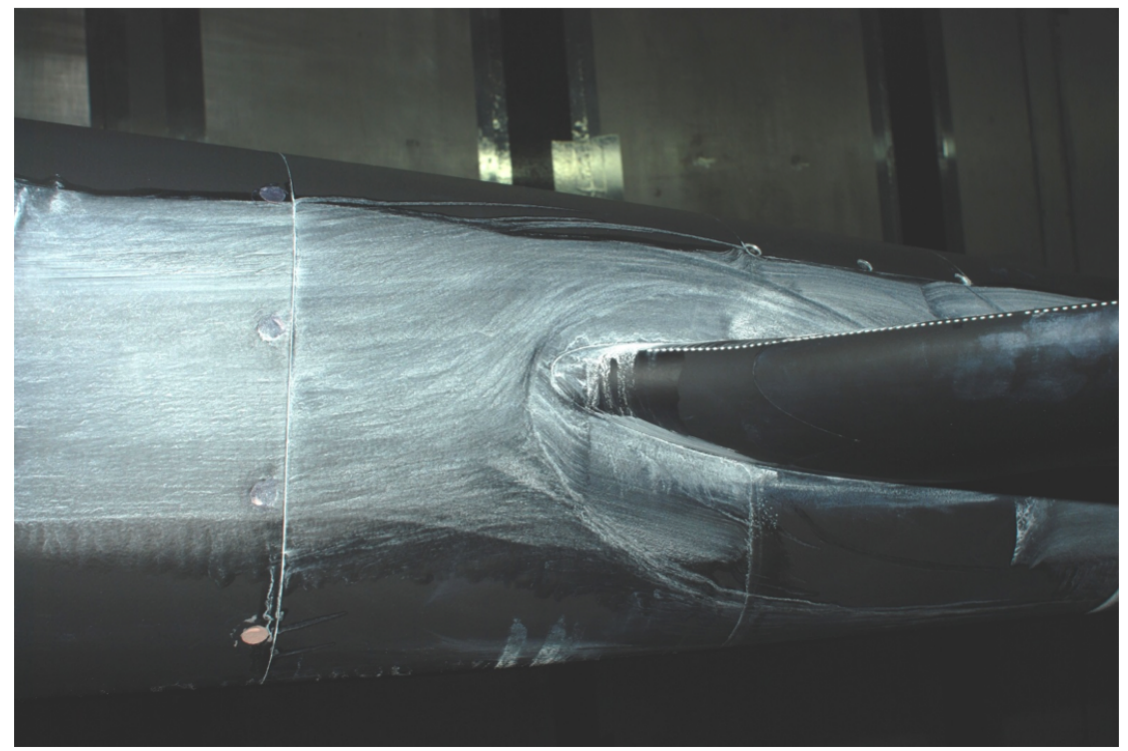

Figure 11. Photograph showing an indication of a horseshoe vortex over the F6 wing at $\alpha=7.5^{\circ}$ in the Virginia Tech tunnel (flow is left to right). 


\section{References}

${ }^{1}$ Vassberg, J. C., Tinoco, E. N., Mani, M., Brodersen, O. P., Eisfeld, B., Wahls, R. A., Morrison, J. H., Zickuhr, T., Laflin, K. R., and Mavriplis, D. J., "Abridged Summary of the Third AIAA Computational Fluid Dynamics Drag Prediction Workshop," Journal of Aircraft, Vol. 45, No. 3, May-June 2008, pp. 781-798.

${ }^{2}$ Levy, D., Laflin, K., Tinoco, E., Vassberg, J., Mani, M., Rider, B., Rumsey, C., Wahls, R., Morrison, J., Broderson, O., Crippa, S., Mavriplis, D., and Murayama, M., "Summary of Data from the Fifth Computational Fluid Dynamics Drag Prediction Workshop," Journal of Aircraft, Vol. 51, No. 4, 2014, pp. 1194-1213.

${ }^{3}$ Sclafani, A. J., Vassberg, J. C., Harrison, N. A., Rumsey, C. L., Rivers, S. M., and Morrison, J. H., "CFL3D/OVERFLOW Results for DLR-F6 Wing/Body and Drag Prediction Workshop Wing," Journal of Aircraft, Vol. 45, No. 3, 2008, pp. 762-780.

${ }^{4}$ Eliasson, P., Nordstrom, J., Peng, S.-H., and Tysell, L., "Effect of Edge-based Discretization Schemes in Computations of the DLR F6 Wing-Body Configuration,” AIAA Paper 2008-4153, June 2008.

${ }^{5}$ Sclafani, A. J., Vassberg, J. C., Winkler, C., Dorgan, A. J., Mani, M., Olsen, M. E., and Coder, J. G., "Analysis of the Common Research Model Using Structured and Unstructured Mashes," Journal of Aircraft, Vol. 51, No. 4, 2014, pp. 1223-1243.

${ }^{6}$ Bordji, M., Gand, F., Deck, S., and Brunet, V., "Investigation of a Nonlinear Reynolds-Averaged Navier-Stokes Closure for Corner Flows," AIAA Journal, article in advance, DOI: 10.2514/1.J054313.

${ }^{7}$ Gand, F., Deck, S., Brunet, V., and Sagaut, P., "Flow Dynamics Past a Simplified Wing Body Junction,” Physics of Fluids, Vol. 22, 2010, 115111.

${ }^{8}$ Wood, D. H. and Westphal, R. V., "Measurements of the Flow Around a Lifting-Wing/Body Junction," AIAA Journal, Vol. 30, No. 1, 1992, pp. 6-12.

${ }^{9}$ Barber, T. J., “An Investigation of Strut-Wall Intersection Losses," Journal of Aircraft, Vol. 15, No. 10, 1978, pp. 676-681.

${ }^{10}$ Gand, F., Brunet, V., and Deck, S., "A Combined Experimental, RANS and LES Investigation of a Wing Body Junction Flow," AIAA Paper 2010-4753, June-July 2010.

${ }^{11}$ Spalart, P. R. and Allmaras, S. R., “A One-Equation Turbulence Model for Aerodynamic Flows,” Recherche Aerospatiale, No. 1, 1994, pp. $5-21$.

${ }^{12}$ Menter, F. R., "Two-Equation Eddy-Viscosity Turbulence Models for Engineering Applications," AIAA Journal, Vol. 32, No. 8, 1994, pp. $1598-1605$.

${ }^{13}$ Yamamoto, K., Tanaka, K., and Murayama, M., "Comparison Study of Drag Prediction for the 4th CFD Drag Prediction Workshop using Structured and Unstructured Mesh Methods," AIAA Paper 2010-4222, June-July 2010.

${ }^{14}$ Spalart, P. R., "Strategies for Turbulence Modelling and Simulation," International Journal of Heat and Fluid Flow, Vol. 21, 2000, pp. 252-263.

${ }^{15}$ Rumsey, C. L., "Application of Reynolds Stress Models to Separated Aerodynamic Flows," in Differential Reynolds Stress Modeling for Separating Flows in Industrial Aerodynamics, ed: B. Eisfeld, Springer Tracts in Mechanical Engineering, Springer International Publishing, Cham, Switzerland, 2015, pp. 19-37.

${ }^{16}$ Dandois, J., "Improvement of Corner Flow Prediction Using the Quadratic Constitutive Relation," AIAA Journal, Vol. 52, No. 12, 2014, pp. 2795-2806.

${ }^{17}$ Gessner, F. B., "The Origin of Secondary Flow in Turbulent Flow Along a Corner," Journal of Fluid Mechanics, Vol. 58, Part 1, 1973, pp. $1-25$.

${ }^{18}$ Chen, H.-C., "Assessment of a Reynolds Stress Closure Model for Appendage-Hull Junction Flows," Journal of Fluids Engineering, Vol. 117, December 1995, pp. 557-563.

${ }^{19}$ Simpson, R. L., "Junction Flows," Annual Review of Fluid Mechanics, Vol. 33, 2001, pp. 415-443.

${ }^{20}$ Olcmen, M. S. and Simpson, R. L., "Some Features of a Turbulent Wing-Body Junction Vortical Flow," AIAA Paper 1997-0651, January 1997.

${ }^{21}$ Devenport, W. J., Simpson, R. L., Dewitz, M. B., and Agarwal, N. K., "Effects of a Leading-Edge Fillet on the Flow Past an Appendage-Body Junction," AIAA Journal, Vol. 30, No. 9, 1992, pp. 2177-2183.

${ }^{22}$ Devenport, W. J. and Simpson, R. L., "Time-Dependent and Time-Averaged Turbulence Structure Near the Nose of a Wing-Body Juncture," Journal of Fluid Mechanics, Vol. 210, 1990, pp. 23-55.

${ }^{23}$ Paik, J., Escauriaza, C., and Sotiropoulos, F., "On the Bimodal Dynamics of the Turbulent Horseshoe Vortex System in a Wing-Body Junction," Physics of Fluids, Vol. 19, 2007, 045107.

${ }^{24}$ Gand, F., Brunet, V., and Deck, S., "Experimental and Numerical Investigation of a Wing Body Junction Flow," AIAA Journal, Vol. 50, No. 12, 2012, pp. 2711-2719.

${ }^{25}$ Gand, F., Monnier, J.-C., Deluc, J.-M., and Choffat, A., "Experimental Study of the Corner Flow Separation on a Simplified Junction," AIAA Journal, Vol. 53, No. 10, 2015, pp. 2869-2877.

${ }^{26}$ Bordji, M., Gand, F., Brunet, V., and Deck, S., "Comparative Study of Linear and Non-Linear RANS Closures for Corner Flows," AIAA Paper 2014-2690, June 2014.

${ }^{27}$ Aeschliman, D. P. and Oberkampf, W. L., "Experimental Methodology for Computational Fluid Dynamics Code Validation," AIAA Journal, Vol. 36, No. 5, 1998, pp. 733-741.

${ }^{28}$ Oberkampf, W. L. and Roy, C. J., Verification and Validation in Scientific Computing, Cambridge University Press, Cambridge UK, 2012.

${ }^{29}$ Shur, M. L., Strelets, M. K., Travin, A. K., and Spalart, P. R., "Turbulence Modeling in Rotating and Curved Channels: Assessing the Spalart-Shur Correction," AIAA Journal, Vol. 38, No. 5, 2000, pp. 784-792.

${ }^{30}$ Cécora, R-D., Radespiel, R., Eisfeld, B., and Probst, A., "Differential Reynolds-Stress Modeling for Aeronautics," AIAA Journal, Vol. 53, No. 3, 2015, pp. 739-755.

${ }^{31}$ Campbell, R. L., "Efficient Viscous Design of Realistic Aircraft Configurations," AIAA Paper 98-2539, June 1998.

${ }^{32}$ Nichols, R. H. and Buning, P. G., "User's Manual for OVERFLOW 2.2, Aug. 2010," http://people.nas.nasa.gov/ pulliam/Overflow/ Overflow_Manuals.html (retrieved Nov. 2015). 
${ }^{33}$ Biedron, R. T., Carlson, J.-R., Derlaga, J. M., Gnoffo, P. A., Hammond, D. P., Jones, W. T., Kleb, B., Lee-Rausch, E. M., Nielsen, E. J., Park, M. A., Rumsey, C. L., Thomas, J. L., and Wood, W. A., "FUN3D Manual: 12.7," NASA/TM-2015-218761, May 2015.

${ }^{34}$ Squire, L. C., "The Motion of a Thin Oil Sheet Under the Steady Boundary Layer on a Body," Journal of Fluid Mechanics, Vol. 11, No. 2, 1961, pp. 161-179. 